\title{
Distribución altitudinal de la riqueza y diversidad de aves en turberas de altura, Costa Rica.
}

\author{
Paola Gastezzi-Arias ${ }^{1,2}$ iD , Daniel Martínez-Araya ${ }^{3}$ iD \& Gabriela Jones-Román ${ }^{4}$ iD \\ 1. Doctorado en Ciencias Naturales para el Desarrollo Costa Rica (DOCINADE), Instituto Tecnológico de Costa Rica, \\ Universidad Nacional, Universidad Estatal a Distancia, Costa Rica, pgastezzi@uned.ac.cr \\ 2. Laboratorio de Vida Silvestre y Salud, Vicerrectoría de Investigación, Universidad Estatal a Distancia, Costa Rica. \\ 3. Unión de Ornitólogos de Costa Rica, Costa Rica, jacamerops@yahoo.com \\ 4. Laboratorio de Ecología Urbana, Vicerrectoría de Investigación de la Universidad Estatal a Distancia, \\ gjones@uned.ac.cr
}

Recibido 30-VIII-2021 - Corregido 11-X-2021 - Aceptado 8-XI-2021

DOI: https://doi.org/10.22458/urj.v13i2.3716

\begin{abstract}
Altitudinal distribution of bird richness and diversity in high altitude peatlands, Costa Rica". Introduction: Highland peatlands play an important role in the maintenance of biodiversity and in the functionality of highland ecological processes. In Costa Rica, information on the richness and diversity of birds in high altitude peatlands is scarce. Objective: To analyze the altitudinal distribution of bird richness and diversity in high altitude peatlands. Methods: We sampled four different altitudinal sites between 2400 to $3100 \mathrm{~m}$. Three observation points were established at each site, each separated by 100 $\mathrm{m}$. The sampling included the dry season, transition to rainy, rainy and transition to dry (September 2018 to Sept. 2019). Sampling hours were between 06:00 and 09:00 and we used two methods: point count and area search. Results: We identified 75 species (25 families, 11 orders), in eight peatlands. We found the highest diversity $\left(H^{\prime}=3,12\right)$ and richness of birds (49 species observed and 56 expected) in peatlands at $2400 \mathrm{~m}$ altitude, and the smallest bird richness at 2600 and $2800 \mathrm{~m}$. We found $43 \%$ of bird species in all gradients to be endemic, $34 \%$ highland residents, and $14 \%$ latitudinal migrants. Conclusions: The high diversity of birds in these high altitude peatlands demonstrates that they are necessary ecosystems for the survival and maintenance of highland endemism. We recommend long-term bird monitoring to detect changes in bird communities, particularly with current climate change.
\end{abstract}

Keywords: biodiversity, wetlands, ecosystems, conservation, community.
RESUMEN. Introducción: Las turberas de altura juegan un papel importante en el mantenimiento de la biodiversidad y funcionamiento de los procesos ecológicos locales. En Costa Rica, la información sobre la riqueza y diversidad de aves en turberas de altura es escasa. Objetivo: Analizar la distribución altitudinal de la riqueza y diversidad de aves en turberas de altura. Métodos: Muestreamos cuatro sitios entre 2400 y 3100 m de altitud. Establecimos tres puntos de observación en cada sitio, separados por $100 \mathrm{~m}$. El muestreo incluyó la estación seca, transición a lluviosa, lluviosa, y transición a seca (setiembre de 2018 a setiembre de 2019). El horario de muestreo fue entre las 06:00 y las 09:00 y utilizamos dos métodos: recuento de puntos $y$ búsqueda en área. Resultados: Identificamos 75 especies (25 familias, 11 órdenes), en ocho turberas. Encontramos la mayor diversidad $\left(H^{\prime}=3,12\right)$ y riqueza de aves (49 especies observadas y 56 esperadas) en turberas a $2400 \mathrm{~m}$ de altitud, y la menor a 2600 y 2800 m. Encontramos que el $43 \%$ de las especies en todos los gradientes son endémicas, el $34 \%$ residentes de y el $14 \%$ migrantes latitudinales. Conclusiones: La gran diversidad de aves en estas turberas de altura demuestra que son ecosistemas necesarios para la supervivencia y mantenimiento del endemismo local. Recomendamos monitorear las aves a largo plazo para detectar cambios en las comunidades de aves, particularmente con el cambio climático actual

Palabras clave: biodiversidad, humedales, ecosistemas, conservación, comunidad. 
En las tierras altas de Costa Rica existen humedales que están representados por cuerpos de agua como ríos, lagunas, lagos y turberas. Estas últimas son importantes para mantener la funcionalidad de los procesos hidrológicos, ecológicos y biológicos de las tierras altas y por ser depósitos de materia orgánica en descomposición, producida por plantas adaptadas a vivir en condiciones de saturación permanente de agua, con reducido contenido de oxígeno y pocos nutrientes (Blanco \& Balze, 2004; León \& Reyes, 2017).

En Costa Rica, las turberas de altura se ubican en la Cordillera de Talamanca, en un gradiente altitudinal que va desde los $2400 \mathrm{~m}$ hasta los $3400 \mathrm{~m}$ aproximadamente. A pesar de su importancia y del hecho de estar protegidas dentro de figuras de conservación que incluyen sitios Ramsar (Cleef \& Chaverri, 1996). Por otra parte, en el país existen vacíos de información ecológica y biológica sobre estos ecosistemas. Las investigaciones sobre turberas realizadas son muy puntuales y se limitan a descripciones de las comunidades vegetales, y generalidades específicas relacionadas a sus características ecológicas (Cleef \& Chaverri, 1996; Bermúdez \& Sánchez 2000).

En países como Chile, Perú, Argentina, Colombia, Ecuador y Bolivia, los páramos, las turberas y los bofedales, han sido estudiados para salvaguardar su biodiversidad por ser ecosistemas frágiles vinculados a las dinámicas hídricas, cumplir funciones como la de acumular carbono, y por prestar servicios ecosistémicos relevantes. En Costa Rica se ha prestado atención y enfocado los esfuerzos de conservación a los páramos, por lo que las turberas de altura han quedado rezagadas de esos esfuerzos, y por ende se hace necesario generar información científica sobre su biodiversidad.

Investigaciones sobre turberas en Latinoamérica, demuestran que son ecosistemas vulnerables a los efectos del cambio en el clima local (Rodríguez \& Granados 2017) y en especial a las perturbaciones antropogénicas (cambio de uso del suelo), lo que podría producir un desequilibrio en el funcionamiento ecosistémico y, como consecuencia, la reducción de la riqueza y diversidad de las especies que habitan en ellas, además una disminución en la calidad de los servicios ecosistémicos que proporcionan (Buytaert et al., 2011).

La evaluación de comunidades biológicas permite conocer la dinámica poblacional y ecológica en un ecosistema, lo cual a su vez conlleva a desarrollar esfuerzos de conservación y favorece la gestión de la biodiversidad. Para esto, existen parámetros como la riqueza y diversidad de especies, muy utilizados en estudios de ecología de comunidades y de biología de la conservación (Loaiza, 2017; Rodríguez \& Granados, 2017).

El uso de indicadores biológicos para monitorear los ecosistemas de altura es clave porque éstos nos dan información del estado actual de los hábitats; información necesaria para diseñar estrategias de protección y para la designación de áreas prioritarias para su conservación y gestión (Rodríguez-Arias \& Silva-Benavides, 2017). En este contexto, las aves han sido utilizadas como indicadores biológicos en muchos estudios por ser un grupo diverso, con una historia natural y ecología bien documentadas, están presentes en varios ecosistemas y se conocen sus patrones de comportamiento en los diferentes ecosistemas donde se encuentran (González et al., 2003; Villegas \& Garitano-Zavala, 2008; Loaiza, 2017; Rodríguez \& Granados, 2017).

Este trabajo tiene como objetivo principal analizar la distribución altitudinal de la riqueza y diversidad de aves en turberas de altura -como ecosistemas de alto valor ecológico aunque poco estudiados en el país- con la finalidad de (i) caracterizar por primera vez la avifauna de las turberas de altura en Costa Rica, (ii) destacar su importancia en función a la diversidad de aves de tierra altas, incluyendo endemismos y (iii) establecer una línea base para el uso futuro estos parámetros como indicadores del estado de conservación de las turberas. 


\section{MATERIALES Y MÉTODOS}

El estudio se realizó en ocho turberas que se localizan en el Parque Nacional Tapantí Macizo de la Muerte $\left(09,55478^{\circ} \mathrm{N},-83,75962^{\circ} \mathrm{W}\right)(\mathrm{T} 1, \mathrm{~T} 2, \mathrm{~T} 3, \mathrm{~T} 4, \mathrm{~T} 5$ y T6), y en la Reserva Biológica Cerro Vueltas $\left(09,62169^{\circ} \mathrm{N},-83,85669^{\circ} \mathrm{W}\right.$ ) (T7 y T8) (Fig. 1). Se seleccionaron cuatro sitios de muestreo con dos turberas por sitio, por su fácil acceso y por presentar cuerpos de agua visibles. Además, fueron ubicados a diferentes altitudes que van desde los 2400 a los $3100 \mathrm{~m}$.
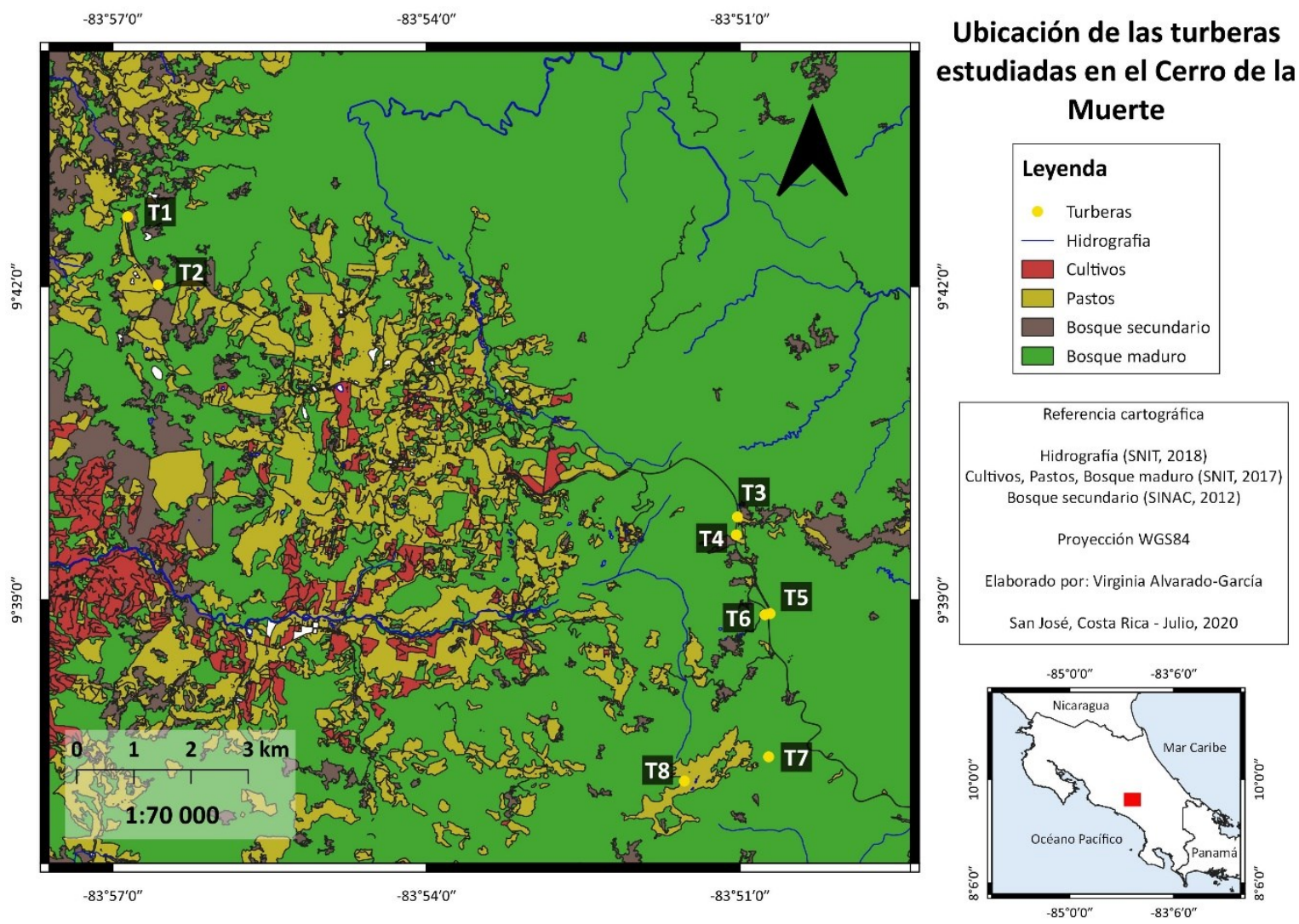

Fig. 1. Ubicación de las turberas de altura en el Cerro de la Muerte.

En cada turbera se establecieron tres puntos de observación separados por $100 \mathrm{~m}$ cada uno (Arévalo, 2014; Kazuya et al., 2014). El muestreo se realizó por el método de conteo por puntos con un radio de observación determinado de $30 \mathrm{~m}$ aproximadamente. Para complementar las observaciones se realizó una búsqueda intensiva dentro de cada turbera. La búsqueda intensiva es un método que consiste en efectuar una serie de conteos meticulosos, donde el observador recorre un área determinada en busca de aves, para aumentar la probabilidad de detección de aquellas especies particularmente inconspícuas o silenciosas (Ralph et al., 1996).

Los muestreos se realizaron entre septiembre del 2018 y septiembre de 2019, se incluyó la estación seca, transición a lluviosa, lluviosa y transición a seca. Las horas del muestreo fue entre las 06:00 a 09:00. En cada punto de conteo se realizó una observación de 10 minutos; así, cada visita, duró 50 minutos en cada turbera, dado que la búsqueda intensiva duró 20 minutos. Se contabilizaron todos los individuos por especie, y registraron todas las aves dentro de los límites de cada punto de conteo; utilizamos binoculares Vortex de 10x40 para las observaciones. Los cuatro sitios de muestreo fueron visitados una vez por mes, realizando un esfuerzo de muestreo de 105,6 horas. 
Para el análisis estadístico, se generaron curvas de acumulación de especies basadas en las especies observadas (Sobs) con el programa Estimates 9.1.0 (Colwell, 2019) con el fin de conocer la riqueza de aves entre los diferentes pisos altitudinales. Con el mismo programa se calculó la riqueza de especies de aves estimada por medio del estimador no paramétrico Bootstrap -es un estimador que arroja resultados más precisos al estimar la riqueza de ensambles con gran cantidad de especies raras- (Colwell et al., 2004; Magurran, 2004).

Se calcularon los índices de riqueza específica, diversidad de Shannon-Wiener y dominancia de Berger-Parker con el programa Species Diversity \& Richness 4.1.2 (Seaby \& Henderson, 2006) para comparar de la diversidad de las aves entre los meses de muestreo, estratos altitudinales y las estaciones climáticas. Además, se calcularon los intervalos de confianza al $95 \%$ de los estimados mediante procedimientos "bootstraping" y se efectuaron pruebas de aleatorización (randomization tests) para realizar comparaciones estadísticas (Solow, 1993).

Para evaluar el grado de recambio de especies entre los ensambles de aves en las diferentes altitudes y las estaciones climáticas, se utilizó el índice de disimilitud de Bray-Curtis con las abundancias relativas de las especies. Los valores obtenidos a partir del índice de disimilitud varían desde cero, cuando dos grupos son idénticos en composición de especies, hasta uno, cuando las especies de ambos grupos son completamente distintas (Colwell \& Coddington, 1994). Para evaluar si existían diferencias estadísticas en entre las diferentes altitudes y estaciones climáticas se realizó un análisis PERMANOVA, junto con análisis de comparaciones múltiples a posteriori. Finalmente, mediante un análisis SIMPER se identificaron a las principales especies responsables de la disimilitud entre la composición de los ensambles de aves en las diferentes altitudes y las estaciones climáticas. Estos análisis se realizaron con el paquete "vegan" en el programa R versión 3.5.2 (Oksanen et al., 2019).

\section{RESULTADOS}

Se registraron un total de 75 especies de aves, pertenecientes a 25 familias y a 11 órdenes (Apéndice digital, Tabla 1). Además, se encontró que el $43 \%$ de las especies en las diferentes altitudes son endémicas, el $34 \%$ residentes de las tierras altas y el $14 \%$ migratorias latitudinales (Fig. 2). Por otro lado, las familias mejor representadas en este trabajo fueron Tyrannidae con un 17\%, Trochilidae $15 \%$, y las familias Hirundinidae, Turdidae, Passerellidae y Parulidae con un $14 \%$ cada una. Por otra parte, la mayoría de las especies de aves son insectívoras (36\%), seguidas por las frugívoras (11\%). Las aves observadas en las diferentes altitudes no son exclusivas de las turberas de altura, pero algunas como las migratorias altitudinales usan estos ecosistemas para suplir sus requerimientos biológicos como alimentación, anidación, reproducción, y refugio.

La mayor riqueza de aves en turberas fue encontrada en el estrato de menor elevación ( 2400 $\mathrm{m}$ ) con 49 especies observadas, y 56 esperadas. Mientras que, en las elevaciones de $2600 \mathrm{~m}$ y 2800 $\mathrm{m}$, las aves presentaron la menor riqueza, tanto observada como la estimada. La eficiencia del muestreo para los estratos altitudinales $2400 \mathrm{~m}, 2600 \mathrm{~m}, 2800 \mathrm{~m}$ y $3100 \mathrm{~m}$, fue de $87,16 \%, 88,34 \%$, $90,90 \%$ y $86,47 \%$, respectivamente. Las curvas de acumulación de especies realizadas con Sobs (Sp Obser) no se estabilizaron (no alcanzaron la asíntota) al igual que la generadas con el estimador Bootstrap (Fig. 3).

Al comparar la riqueza entre turberas a diferentes altitudes, se observó la mayor riqueza de especies a los 2400 m (49 especies), y la menor a los 2800 m (36 especies). Según los análisis de diversidad (índices de Shannon) y dominancia (índice de Berger-Parker), hubo diferencias significativas entre turberas de diferentes altitudes: $2400 \mathrm{~m}(\mathrm{H}=3,12), 2600 \mathrm{~m}(\mathrm{H}=2,86)$ y $2800 \mathrm{~m}$ $(H=2,91)$. Mientras que, la diversidad y dominancia fue similar entre turberas de los $2400 \mathrm{~m}(\mathrm{H}=3,12)$ y las de los $3100 \mathrm{~m}(\mathrm{H}=3,08)$ (Tabla 2$)$. 

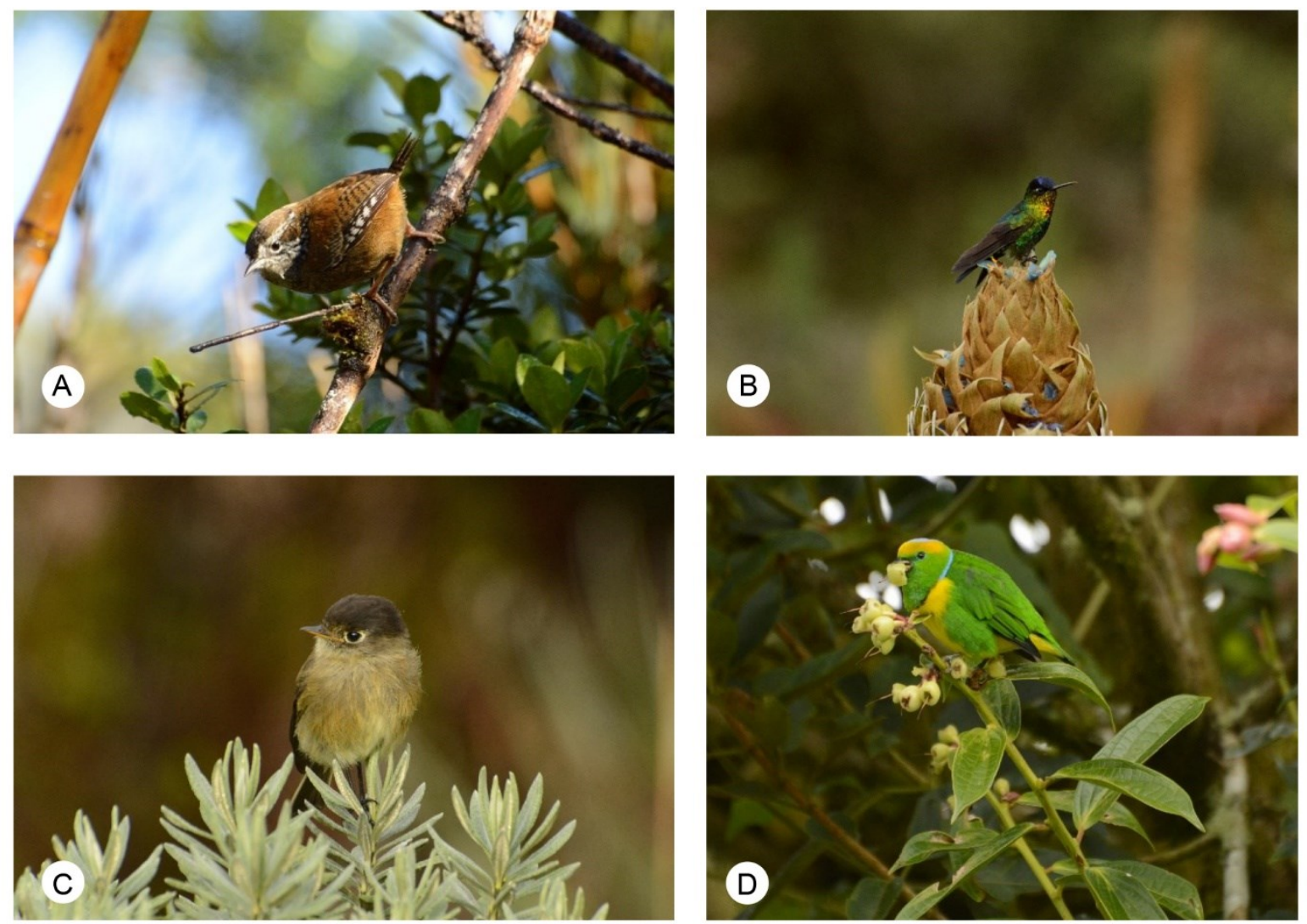

Fig. 2. Especies de aves endémicas representativas de tierras altas de Costa Rica y presentes en las turberas de altura del Cerro de la Muerte. A. Thryorchilus browni; B. Panterpe insignis; C. Empidonax atriceps; D. Chlorophonia callophrys. Fotos: Daniel Martínez-Araya.

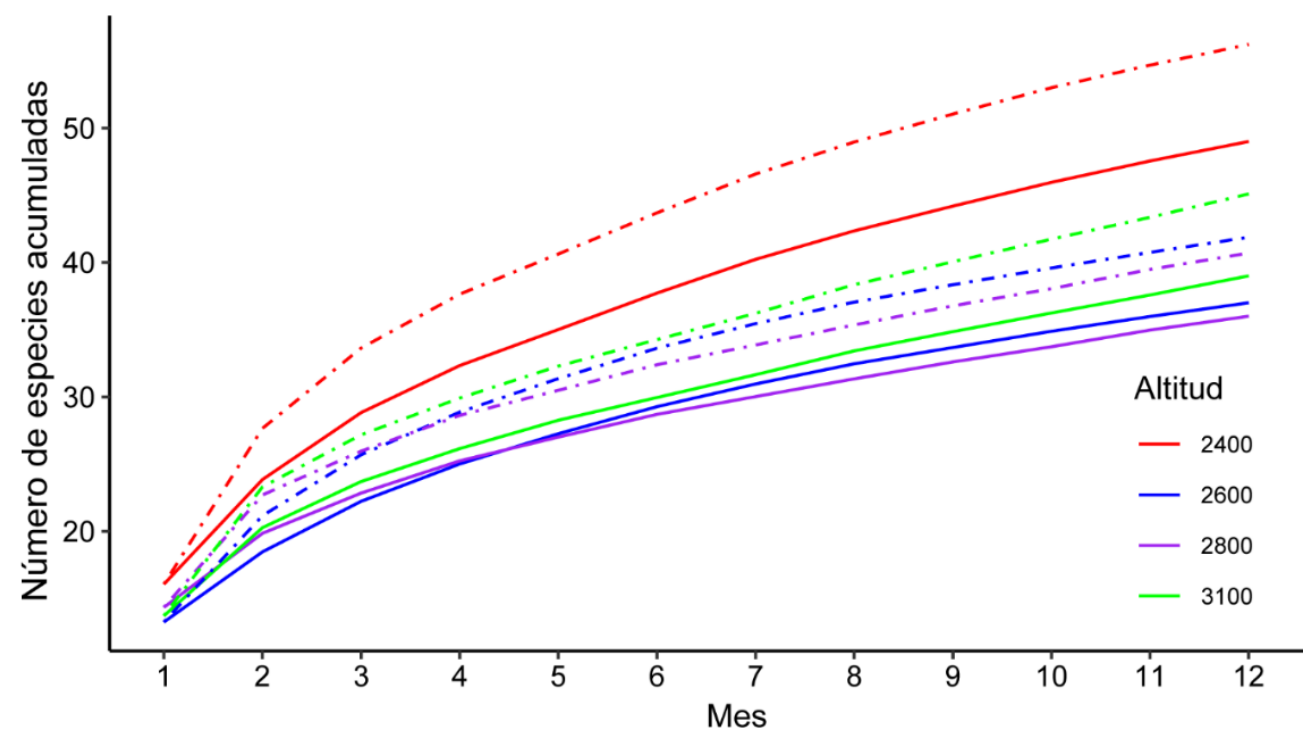

Fig. 3. Curvas de acumulación de la riqueza de especies de aves (líneas continuas) y comparación con la riqueza esperada por el estimador no paramétrico Bootstrap (líneas punteadas) en función del esfuerzo acumulativo de muestreo (meses) en turberas a diferentes altitudes en el Cerro de la Muerte, 2018- 2019. 
TABLA 2

Comparación de los índices de diversidad de aves en las turberas de altura en el Cerro de la Muerte 2018 -2019.

\begin{tabular}{lcccc}
\hline \multicolumn{1}{c}{ Índice } & $\mathbf{2 4 0 0}$ & $\mathbf{2 6 0 0}$ & $\mathbf{2 ~ 8 0 0}$ & $\mathbf{3 1 0 0}$ \\
\hline Riqueza de especies & 49 & 37 & 36 & 39 \\
Índice de Shannon & $3,12^{\mathrm{a}}$ & $2,86^{\mathrm{b}}$ & $2,91^{\mathrm{b}}$ & $3,08^{\mathrm{a}}$ \\
& $(3,04-3,16)$ & $(2,74-2,91)$ & $(2,79-2,96)$ & $(2,95-3,11)$ \\
& $0,13^{\mathrm{a}}$ & $0,18^{\mathrm{b}}$ & $0,20^{\mathrm{b}}$ & $0,12^{\mathrm{a}}$ \\
Índice de dominancia Berger-Parker & $(0,12-0,16)$ & $(0,15-0,21)$ & $(0,16-0,23)$ & $(0,10-0,15)$ \\
& & & & $\mathbf{2 8 0 0}$ \\
\hline Coeficiente de disimilitud (Bray-Curtis) & $\mathbf{2 4 0 0}$ & $\mathbf{2 6 0 0}$ & & \\
\hline 2600 & 0,27 & & & \\
2800 & 0,35 & 0,24 & 0,35 \\
3100 & 0,44 & 0,37 & & \\
\hline
\end{tabular}

* Las letras distintas indican diferencias significativas de las pruebas de aleatorización ( $p<0.05)$.

Según el análisis PERMANOVA hay diferencias en la composición de especies entre los ensambles de aves presentes en turberas a diferentes altitudes $(F=5,39 ; p=0,001)$ y estaciones climáticas $(F=3,82 ; p=0,001)$. Adicionalmente, según el índice de Bray-Curtis hay una mayor disimilitud de los ensambles de aves entre los $2400 \mathrm{~m}$ y $3100 \mathrm{~m}(0,44)$ con una diferencia significativa $(F=7,76 ; p=0,006)$ (Tabla 3 ).

\section{TABLA 3}

Análisis del modelo PERMANOVA de la distancia Bray-Curtis según el rango altitudinal y estación climática, en turberas de altura en el Cerro de la Muerte 2018 -2019

\begin{tabular}{lcccccc}
\hline \multicolumn{1}{c}{ Variable } & Df & SumsOfSqs & MeanSqs & F.Model & $\mathbf{R}^{\mathbf{2}}$ & $\mathbf{P}$ \\
\hline Altitud & 3 & 1,84 & 0,61 & 5,39 & 0,24 & 0,001 \\
Estación climática & 3 & 1,31 & 0,44 & 3,82 & 0,17 & 0,001 \\
Total & 47 & 7,81 & & & 1,00 & \\
\hline
\end{tabular}

Por otro lado, según el índice de Bray-Curtis existe una mayor disimilitud de los ensambles de aves entre la estación seca y lluviosa ( $F=4,91 ; p=0,006)$. Mientras que entre la época seca y transición a lluviosa hay mayor similitud ( $F=2,19 ; p=0,198)$. El análisis SIMPER para los ensambles de aves, identificó quince especies de aves como las responsables del $70 \%$ de su diferencia, entre ellas el Pinchaflor plomizo (Diglossa plumbea), Elainia montañera (Elaenia frantzii) y Paloma collareja (Patagioenas fasciata). Además, el análisis SIMPER determinó que, en turberas a los $2400 \mathrm{~m}$, las especies dominantes fueron el Soterrey de selva pechigris (Henicorhina leucophrys), D. plumbea y Tangara de monte cejiblanca (Chlorospingus pileatus), mientras que, en turberas de los $3100 \mathrm{~m}$, fueron el Mirlo negruzco (Turdus nigrescens) y Chispita volcanera (Selasphorus flammula). 


\section{DISCUSIÓN}

Las curvas de acumulación de especies reflejaron que la cantidad de muestreos realizados no fueron suficientes para observar la totalidad de las especies de aves durante el periodo de muestreo. Una de las causas de este resultado, es que los muestreos se realizaron en las horas de la mañana, dado que en las tardes las condiciones climáticas del área de estudio no lo permitieron por la presencia de lluvias, fuertes vientos y bajas temperaturas. A pesar de que no se alcanzó la asíntota, se realizó un esfuerzo de muestreo bastante alto (105,6 horas de observación) y se cubrieron todos los meses del año.

La mayor riqueza de aves estimada fue en las turberas de los $2400 \mathrm{~m}$. Barrantes (2005), menciona que en Costa Rica la riqueza de aves de tierras altas y de bosques adyacentes disminuye al aumentar la altitud, y alcanza su punto mínimo en el páramo a $3800 \mathrm{~m}$. Al comparar la diversidad de aves entre turberas a diferentes altitudes, se evidencia que existen diferencias estadísticamente significativas entre las turberas a los 2400,2600 y $2800 \mathrm{~m}$. Una de las posibles razones de este resultado es que, en los $2400 \mathrm{~m}$ las aves poseen mayor disponibilidad de recursos alimenticios en especial para las aves de hábitos alimenticos más generalistas que utilizan insectos y frutas en su dieta, a diferencia de las otras turberas donde existe un mosaico continuo de bosque de altura (premontano, montano y páramo) y las aves son más especialistas en sus dietas.

También, se encontraron diferencias en la diversidad de aves entre las turberas del rango altitudinal más alto (3 $100 \mathrm{~m}$ ), con las turberas de los rangos altitudinales intermedios (2 $600 \mathrm{~m} \mathrm{y}$ $2800 \mathrm{~m}$ ), para ambos índices (Shannon y dominancia de Berger-Parker). Esto concuerda con lo reportado por Terborgh (1971), y Martínez \& Rechberger (2007) donde la diversidad de especies de aves cambia gradualmente desde los 1850 hasta los 3170 m, debido a los cambios en la composición de la vegetación con respecto a la altura, lo que podría estar reflejado en los recursos presentes en cada uno de los rangos altitudinales.

Por otra parte, Terborgh (1971), y Blake \& Loiselle (2000) indican que, la diversidad de aves en un rango altitudinal muestra una disminución de la riqueza de aves con respecto al aumento de la elevación y se manifiesta por la disminución de recursos disponibles y por las transformaciones de las condiciones ambientales. Blake y Loiselle (2000), y Loaiza (2017) mencionan que, las comparaciones entre diferentes altitudes podrían ser de importancia para detectar el recambio de especies en las diferentes elevaciones. Sin embargo, en este trabajo se encontró que existe similitud entre la diversidad de aves de las turberas a los $2400 \mathrm{~m}(\mathrm{H}=3,12)$ y las de los $3100 \mathrm{~m}(\mathrm{H}=3,08)$, y puede ser debido a que existe una fuerte asociación entre grupos de aves y los diversos tipos de vegetación donde cada grupo presenta diferentes ensambles de especies a lo largo de un rango altitudinal.

Según los resultados, la presencia de las aves endémicas registradas en turberas a diferentes altitudes no estuvo determinada por la variación altitudinal. Las turberas se ubican a diferentes altitudes y la distancia entre ellas es de pocos metros aproximadamente $(500 \mathrm{~m}$ a $1 \mathrm{~km}$ ), permitiendo que las aves puedan desplazarse entre las franjas de la vegetación continua que existen en estos rangos de altitud. Esto se explica con lo reportado por Terborgh (1971), y Blake \& Loiselle (2000), que a altitudes similares o con pocas diferencias en rangos altitudinales, no se puede evidenciar asertivamente la transición de aves de una zona de mediana elevación con mayor diversidad.

La estacionalidad climática en el Cerro de la Muerte es bien marcada, en este trabajo se encontró mayor disimilitud de los ensambles de aves entre la estación seca y lluviosa, debido a que en la estación seca la falta de agua en las turberas y los cambios en la fenología de la vegetación, posiblemente reducen los recursos alimenticios y algunas especies de aves realizan desplazamientos entre los diferentes rangos altitudinales en busca de recursos. Las variaciones del paisaje en función de las precipitaciones y la temperatura podrían influir en la disponibilidad de alimento, existiendo 
una variación significativa de la abundancia de aves entre los periodos seco y lluvioso, con un mayor número de aves durante el periodo de lluvias (Peña-Villalobos et al. 2012).

Las especies $H$. leucophrys, $D$. plumbea y $C$. pileatus, fueron dominantes en términos de abundancia a los $2400 \mathrm{~m}$, mientras que, en el gradiente de los $3100 \mathrm{~m}$, fueron dominantes $T$. nigrescens y $S$. flammula, lo que sugiere que estas especies poseen niveles tróficos diversos y a su vez poseen alta representatividad en estos gradientes, lo que evidencia la heterogeneidad de las turberas de altura. Esto concuerda con la distribución altitudinal de las especies de aves en Costa Rica, es así que la distribución de H. leucophrys, va desde los $800 \mathrm{~m}$ hasta los $3800 \mathrm{~m}$, D. plumbea, desde $1200 \mathrm{~m}$ a los $3800 \mathrm{~m}$, S. flammula desde $1200 \mathrm{~m}$ hasta los $3800 \mathrm{~m}$. T. nigrescens desde los 2 $400 \mathrm{~m}$ a los $3800 \mathrm{~m}$, y C. pileatus desde los $2000 \mathrm{~m}$ hasta los $3800 \mathrm{~m}$. Vale la pena destacar que $T$. nigrescens, $C$. pileatus, D. plumbea, S. flammula, son endémicos de tierras altas de Costa Rica y el oeste de Panamá (Stiles y Skutch, 1989; Garrigues, 2014).

En las turberas se identificaron especies de aves perteneciente a diferentes gremios tróficos, la mayoría fueron insectívoras (36\%), seguidas por las frugívoras (11\%) las cuales se observaron alimentándose de frutos de plantas de las familias Ericaceae, Rubiaceae, Onagraceae, entre otras. Es importe mencionar que se observó al Junco paramero (Junco vulcani) y al migratorio Andarríos solitario (Tringa solitaria) alimentándose de insectos acuáticos a la orilla de una de las turberas. Por lo que es importante destacar que las turberas altura proveen de recursos alimenticos, refugio y sitios de descanso para muchas especies de aves.

Sandoval \& Sánchez (2014), mencionan que en Costa Rica existen un total de 85 especies de aves de alta montaña, de las cuales 37 se consideran endémicas de tierras altas de Costa Rica y el oeste de Panamá, y representan aproximadamente el 9,4\% del total de especies del país. Durante esta investigación se registraron 33 especies endémicas de tierras altas y de distribución reducida, que utilizan las turberas, y las zonas de transición entre el bosque y el páramo, por lo que se puede argumentar que las turberas son ecosistemas para la supervivencia y mantenimiento del endemismo que existe en las tierras altas de la zona.

El uso de las aves como indicadoras nos permitió obtener a lo largo de todo el año de muestreo resultados fiables de especies relativamente comunes, endémicas y con distribución restringida de tierras altas, además de detectar especies con alta sensibilidad a los cambios ambientales en el ecosistema como el J. vulcani, Zeledonia coronata, Scytalopus argentifrons y $T$. browni que requieren hábitos específicos del hábitat donde viven (bosque montano y páramo). Lo que podemos afirmar que las turberas brindan esos requerimientos y desempeñan un papel importante para la conservación de las aves, y soportan la funcionalidad de los procesos ecológicos en tierras altas además que proveen los recursos y elementos del hábitat para este grupo de vertebrados.

La carencia de estudios similares en turberas de altura dificulta la comparación de nuestros resultados, por lo que se desconoce si estos siguen un patrón similar en otras turberas de Costa Rica y Latinoamérica. Se concluye que las turberas de la Cordillera de Talamanca albergan por lo menos 75 especies de aves, con un $47 \%$ de endemismo lo que demuestra que estos ecosistemas son de vital importancia para la conservación para las aves de tierras altas y las migratorias latitudinales.

Los resultados justifican la conservación y mayor protección de ecosistemas frágiles como las turberas y es necesario evitar que sufran contaminación, quema o extracción de la flora y fauna. Además, se espera que esta investigación sirva de referencia para el análisis de la riqueza y diversidad de aves en ecosistemas similares al de este estudio. Por lo que, se sugiere realizar estudios que contemplen un análisis ecológico del hábitat donde se encuentran las turberas y realizar monitoreos de aves en tierras altas a largo plazo para detectar cambios en las comunidades de aves y su relación al cambio climático. 


\section{AGRADECIMIENTOS}

Extendemos nuestro agradecimiento a la Universidad Estatal a Distancia por el aval para llevar a cabo este proyecto; así como al Sr. Rodolfo Jiménez y al Sr. Rafael Romero (Don Rafa), por darnos apoyo y permiso para acceder a los sectores de su propiedad durante el trabajo de campo. Al Parque Nacional Los Quetzales por su colaboración en las giras de campo. Además, nuestro agradecimiento especial a Johnny Villareal Orias, por revisar este trabajo, y darnos su apoyo científico, y a Virginia Alvarado-García por la ayuda en la elaboración del mapa. Por otro lado, agradecemos también a los revisores de este trabajo por sus valiosos aportes.

\section{ÉTICA, CONFLICTO DE INTERESES Y DECLARACIÓN DE FINANCIAMIENTO}

Los autores declaramos haber cumplido con todos los requisitos éticos y legales pertinentes, tanto durante el estudio como en la preparación de este documento; que no hay conflictos de interés de ningún tipo, que todas las fuentes financieras se detallan plena y claramente en la sección de agradecimientos. Asimismo, estamos de acuerdo con la versión editada final de esta publicación. El respectivo documento legal firmado se encuentra en los archivos de la revista. La declaración de contribución de cada autor es la siguiente: PGA y DMA toma de datos; PGA y GJR concepción y diseño del manuscrito, PGA, DMA y GJR diseño de metodología y revisión del manuscrito, PGA análisis de datos y escritura final del manuscrito.

\section{REFERENCIAS}

Arévalo, E. (2014). Comparación de métodos de conteo por puntos y de bioacústica para el monitoreo de aves. School for Field Studies y Escuela de Biología, Universidad de Costa Rica. IV Congreso de Ornitología. https://www.researchgate.net/publication/273350590

Barrantes, G. (2005). Aves de los páramos de Costa Rica. En: M. Kapelle \& Horn, SP. (Eds.), Paramos de Costa Rica (pp.521532). Editorial INBio.

Blanco, D.E., \& De la Balze, V.M. (Eds.). (2004). Los Turbales de la Patagonia: Bases para su inventario y la conservación de su biodiversidad. Publicación No. 19. Wetlands International.

Blake, J.G., \& Loiselle, B.A. (2000). Diversity of birds along an elevational gradient in the Cordillera Central, Costa Rica. Revista Auk, 117(3), 663-686. https://doi.org/10.1093/auk/117.3.663

Bermúdez, M., \& Sánchez, J. (Eds.). (2000). Identificación de vacíos de información Botánica en Centroamérica. Ediciones WWF - Centroamérica. San José, Costa Rica. https://bit.ly/3ozbvQc

Buytaert, W., Cuesta-Camacho, F., \& Tobón, C. (2011). Potential Impacts of Climate Change on the Environmental Services of Humid Tropical Alpine Regions. Global Ecology and Biogeography, 20(1), 19-33. https://doi.org/10.1111/j.1466$\underline{8238.2010 .00585 . x}$

Colwell, R., \& Coddington, J. (1994). Estimating terrestrial biodiversity through extrapolation. The Royal Society Publishing, B 345, 101-118. https://doi.org/10.1098/rstb.1994.0091

Colwell, R. K., Chang, X. M., \& Chang, J. (2004). Interpolating, extrapolating, and comparing incidence-based species accumulation curves. Ecology, 98, 2717-2727. https://doi.org/10.1890/03-0557

Colwell, R. (2019). EstimateS: statistical estimation of species richness and shared species from samples, version 9.1.0. http://viceroy.eeb.uconn.edu/estimates. 
Cleef, A., \& Chaverri, A. (1996). Turberas de la Cordillera de Talamanca, Costa Rica. En: Ficha Técnica Turberas de Talamanca. Programa Nacional de Humedales SINAC-MINAE.

Garrigues, R. (2014). The Birds of Costa Rica: A Field Guide (Zona Tropical Publications). Cornell University Press.

González-Ortega, M.A., Guzmán-Hernández, J., Martin-Gómez M.F., \& Domínguez-Velázquez, L.E. (2003). Un método para la selección de aves bioindicadoras con base en sus posibilidades de monitoreo. Huitzil Revista Mexicana de Ornitología, 4(2), 10-16.

Kazuya, N., Landívar, C.M, \& Gómez, M.I. (2014). Monitoreo de las aves para detectar el cambio de la calidad ecosistémica en los bofedales alto andinos. Revista Ecología en Bolivia, 49(3), 73-83. http://www.scielo.org.bo/pdf/reb/v49n3/v49n3 a07.pdf

Loaiza, C. (2017). Dinámica temporal y espacial de la comunidad de aves en un gradiente altitudinal de la Cordillera Volcánica Central de Costa Rica, vertiente Caribe. Revista Caldasia, 39(2), 310. https://dx.doi.org/10.15446/caldasia.v39n2.60647

León-Alfaro, Y., \& Reyes-Chaves, J. (2018). Delimitación y descripción biofísica de dos turberas y de una laguna endorreica en el sector Salsipuedes-Cerro Asunción, Parque Nacional Tapantí - Macizo de la Muerte, Costa Rica. UNED Research Journal. 10(1), 210-215. https://doi.org/10.22458/urj.v10i1.2030

Magurran, A.E. (2004). Measuring Ecological Diversity. Blackwell Publishing.

Martínez, O., \& Rechberger, J. (2007). Características de la avifauna en un gradiente altitudinal de un bosque nublado andino en La Paz, Bolivia. Revista Peruana de Biología, 14(2), 225-236. https://doi.org/10.15381/rpb.v14i2.1745

Oksanen, J., Blanchet, F.G., Friendly, M., Kindt, R., Legendre, P., McGlinn, D., Minchin, P.R, O'Hara, R.B., Simpson, G.L, Solymos, P., Stevens, M.H.H., Szoecs, E., \& Wagner, H. (2019). Vegan: Community Ecology Package. R Package Version 2.5-4. http://CRAN.R-project.org/package=vegan.

Peña-Villalobos, I., Fibla, P., Salazar, J.E, \& Sallaberry, M. (2012). Cambios temporales en la abundancia y composición del ensamble de aves acuáticas en tranques artificiales en Chile Central. Revista Gayana, 76(2), 92-101. https://dx.doi.org/10.4067/S0717-65382012000300002

Ralph, C.J., Geupel, G.R., Pyle, P., Martin, T.E., DeSante, D.F., \& Mila, B. (1996). Manual de métodos de campo para el monitoreo de aves terrestres. General Technical Report (GTR). PSW-GTR-159. Albany, CA: Pacific Southwest Section, Forest Service, U.S. Department of Agriculture. https://doi.org/10.2737/PSW-GTR-159

Rodríguez, C., \& Granados, I. (2017). Diversidad de aves de la Reserva Biológica Alberto Manuel Brenes y su área de amortiguamiento, Costa Rica. Revista Pensamiento Actual, 17(1), 74-95.

Rodríguez-Arias, C., \& Silva-Benavides, A.M. (2017). Los Humedales de la Quebrada Estero en San Ramón, Costa Rica: importancia y estado actual. Posgrado y Sociedad Revista Electrónica del Sistema de Estudios de Posgrado, 15(1), 13-26. https://doi.org/10.22458/rpys.v15i1.1822

Sandoval, L., \& Sánchez, C. (2014). Lista de aves de Costa Rica: décima octava actualización. Unión de Ornitólogos de Costa Rica. http://uniondeornitologos.com/wp-content/uploads/2014/07/Lista-de-Aves-de-Costa-Rica-XVIII.pdf

Seaby, R.M., \& Henderson, P.A. (2006). Species Diversity and Richness Version 4. Pisces Conservation Ltd. http://www.pisces-conservation.com/ecomhelp/index.html?speciesdiv.htm

Solow, A.R. (1993). A simple test for change in community structure. Journal Animal Ecology, 62(1), $191-193$. https://doi.org/10.2307/5493

Stiles, F.G., \& Skutch, A.F. (1989). A guide to the birds of Costa Rica. Cornell University Press. 
Terborgh, J. (1971). Distribution on environmental gradients: Theory and a preliminary interpretation of distributional patterns in the avifauna of the Cordillera Vilcabamba, Peru. Ecology, 52, 23-40. https://doi.org/10.2307/1934735

Villegas, M., \& Garitano-Zavala, A. (2008). Las comunidades de aves como indicadores ecológicos para programas de monitoreo ambiental en la ciudad de La Paz, Bolivia. Revista Ecología en Bolivia, 43(2), 146-153. https://ecologiaenbolivia.com/documents/Villegas432.pdf 


\section{APÉNDICE DIGITAL}

Tabla 1

Listado de las especies de aves identificadas y observadas en las turberas de altura del Cerro de la Muerte, Costa Rica.

\begin{tabular}{|c|c|c|c|c|}
\hline Familia & Especie & Nombre común & $\begin{array}{c}\text { Condición de } \\
\text { la especie }\end{array}$ & $\begin{array}{l}\text { Grupo } \\
\text { trófico }\end{array}$ \\
\hline Odontophoridae & Dendrortyx leucophrys & Perdiz montañera/ chirrascuá & $\mathrm{R}$ & $\mathrm{F}, \mathrm{I}, \mathrm{G}$ \\
\hline Columbidae & Patagioenas fasciata & Paloma collareja & $\mathrm{R}$ & $\mathrm{F}$ \\
\hline Apodidae & Streptoprocne zonaris & Vencejón collarejo & $\mathrm{R}$ & 1 \\
\hline \multirow[t]{7}{*}{ Trochilidae } & Colibri cyanotus & Colibrí orejivioláceo verde & $\mathrm{R}$ & $\mathrm{N}, \mathrm{I}$ \\
\hline & Eugenes spectabilis & Colibrí magnífico & END & $\mathrm{N}, \mathrm{I}$ \\
\hline & Panterpe insignis & Colibrí garganta de fuego & END & $\mathrm{N}, \mathrm{I}$ \\
\hline & Lampornis castaneoventris & Colibrí montañes Gorgiblanco & END & $\mathrm{N}, \mathrm{I}$ \\
\hline & Lampornis sp. & Colibrí montañes & END & $\mathrm{N}, \mathrm{I}$ \\
\hline & Selasphorus flammula & Colibrí chispita volcanera & END & $\mathrm{N}, \mathrm{I}$ \\
\hline & Selasphorus scintilla & Colibrí chispita gorginaranja & END & $\mathrm{N}, \mathrm{I}$ \\
\hline Scolopacidae & Tringa solitaria & Andarríos solitario & M & $\mathrm{C}, \mathrm{I}$ \\
\hline \multirow[t]{2}{*}{ Cathartidae } & Coragyps atratus & Zopilote negro & $\mathrm{R}$ & $\mathrm{O}, \mathrm{C}$ \\
\hline & Cathartes aura & Zopilote cabecirrojo & $R, M$ & $\mathrm{O}, \mathrm{C}$ \\
\hline \multirow[t]{5}{*}{ Accipitridae } & Elanoides forficatus & Elanio tijereta & $R, M$ & $\mathrm{~F}, \mathrm{I}$ \\
\hline & Accipiter bicolor & Gavilán bicolor & $\mathrm{R}$ & C \\
\hline & Buteo swainsoni & Gavilán de swainson & M & C \\
\hline & Buteo platypterus & Gavilán aludo & M & $\mathrm{C}, \mathrm{I}$ \\
\hline & Buteo jamaicensis & Gavilán colirrojo & $R, M$ & C \\
\hline Trogonidae & Pharomachrus mocinno & Quetzal & $\mathrm{R}$ & $F, I, C$ \\
\hline \multirow[t]{2}{*}{ Picidae } & Melanerpes formicivorus & Carpintero careto & $\mathrm{R}$ & $\mathrm{F}$ \\
\hline & Dryobates villosus & Carpintero serranero & $\mathrm{R}$ & $\mathrm{F}, \mathrm{I}$ \\
\hline Falconidae & Falco rufigularis & Halcón cuelliblanco & $\mathrm{R}$ & C \\
\hline \multirow[t]{2}{*}{ Psittacidae } & Pyrrhura hoffmanni & Perico aliazufrado & END & $\mathrm{F}$ \\
\hline & Bolborhynchus lineola & Perico listado & $\mathrm{R}$ & $\mathrm{F}$ \\
\hline Rhinocryptidae & Scytalopus argentifrons & Tapaculo & END & 1 \\
\hline \multirow[t]{2}{*}{ Furnariidae } & Pseudocolaptes lawrencii & Trepamusgo cachetón & $\mathrm{R}$ & 1 \\
\hline & Margarornis rubiginosus & Subepalo rojizo & END & I \\
\hline \multirow[t]{8}{*}{ Tyrannidae } & Elaenia frantzii & Elainia montañera & $\mathrm{R}$ & $\mathrm{F}$ \\
\hline & Zimmerius parvus & Mosquerito Cejigrís & $\mathrm{R}$ & $N, F$ \\
\hline & Myiodynastes hemichrysus & Mosquero vientridorado & END & $F, I$ \\
\hline & Contopus cooperi & Pibí boreal & M & 1 \\
\hline & Contopus lugubris & Pibí sombrío & END & I \\
\hline & Contopus sordidulus & Pibí occidental & $M, R ?$ & I \\
\hline & Contopus sp. & Mosquerito & $M, R ?$ & I \\
\hline & Empidonax atriceps & Mosquerito cabecinegro & END & I \\
\hline \multirow[t]{2}{*}{ Vireonidae } & Cyclarhis gujanensis & Vireón cejirrufo & $\mathrm{R}$ & 1 \\
\hline & Vireo carmioli & Vireo aliamarillo & END & $\mathrm{F}$ \\
\hline
\end{tabular}




\begin{tabular}{|c|c|c|c|c|}
\hline \multirow[t]{6}{*}{ Hirundinidae } & Riparia riparia & Golondrina ribereña & M & I \\
\hline & Pygochelidon cyanoleuca & Golondrina azul y blanco & $\mathrm{R}, \mathrm{M}$ & I \\
\hline & Progne subis & Martín purpúrea & M & I \\
\hline & Hirundo rustica & Golondrina tijereta & M & I \\
\hline & Petrochelidon pyrrhonota & Golondrina risquera & M & I \\
\hline & Petrochelidon fulva & Golondrina cavernícola & $A$ & I \\
\hline \multirow[t]{3}{*}{ Troglodytidae } & Troglodytes ochraceus & Soterrey ocroso & END & I \\
\hline & Thryorchilus browni & Soterrey de bambú & END & I \\
\hline & Henicorhina leucophrys & Soterrey de selva pechigrís & $\mathrm{R}$ & I \\
\hline \multirow[t]{6}{*}{ Turdidae } & Myadestes melanops & Jilguero & END & $\mathrm{F}$ \\
\hline & Catharus gracilirostris & Zorzal piquinegro & END & I \\
\hline & Catharus frantzii & Zorzal gorrirojizo & $\mathrm{R}$ & I \\
\hline & Turdus nigrescens & Mirlo negruzco & END & $\mathrm{F}, \mathrm{I}$ \\
\hline & Turdus grayi & Yiguirro & $\mathrm{R}$ & $\mathrm{F}, \mathrm{I}, \mathrm{C}$ \\
\hline & Turdus plebejus & Mirlo montañero & $\mathrm{R}$ & $\mathrm{F}, \mathrm{I}$ \\
\hline \multirow[t]{2}{*}{ Ptilogonatidae } & Phainoptila melanoxantha & Capulinero negro y amarillo & END & $\mathrm{F}, \mathrm{I}$ \\
\hline & Ptiliogonys caudatus & Capulinero colilargo & END & $\mathrm{F}, \mathrm{I}$ \\
\hline \multirow[t]{3}{*}{ Fringillidae } & Chlorophonia callophrys & Clorofonia cejidorada & END & $\mathrm{F}, \mathrm{I}$ \\
\hline & Chlorophonia elegantissima & Eufonia capuchiceleste & $\mathrm{R}$ & $\mathrm{F}$ \\
\hline & Spinus xanthogastrus & Jilguero vientriamarillo & $\mathrm{R}$ & $F, I$ \\
\hline \multirow[t]{6}{*}{ Passerellidae } & Chlorospingus pileatus & Tangara de monte cejiblanca & END & $\mathrm{F}, \mathrm{I}$ \\
\hline & Chlorospingus flavopectus & Tangara de monte ojeruda & $\mathrm{R}$ & $F, I$ \\
\hline & Junco vulcani & Junco paramero & END & $\mathrm{F}, \mathrm{I}, \mathrm{G}$ \\
\hline & Zonotrichia capensis & Comemaíz & $\mathrm{R}$ & $\mathrm{F}, \mathrm{I}, \mathrm{G}$ \\
\hline & Pezopetes capitalis & Saltón patigrande & END & $\mathrm{F}, \mathrm{I}, \mathrm{G}$ \\
\hline & Atlapetes tibialis & Saltón de muslos amarillos & END & $\mathrm{F}, \mathrm{I}, \mathrm{N}$ \\
\hline Zeledoniidae & Zeledonia coronata & Zeledonia & END & I \\
\hline \multirow[t]{6}{*}{ Parulidae } & Oreothlypis gutturalis & Reinita garganta de fuego & END & $\mathrm{I}, \mathrm{F}$ \\
\hline & Setophaga virens & Reinita cariamarilla & M & 1 \\
\hline & Basileuterus melanogenys & Reinita carinegra & END & $\mathrm{I}, \mathrm{F}$ \\
\hline & Cardellina pusilla & Reinita gorrinegra & M & I \\
\hline & Myioborus miniatus & Candelita pechinegra & $\mathrm{R}$ & I \\
\hline & Myioborus torquatus & Candelita collareja & END & 1 \\
\hline \multirow[t]{2}{*}{ Cardinalidae } & Piranga bidentata & Tangara dorsirrayada & $\mathrm{R}$ & $\mathrm{I}, \mathrm{F}$ \\
\hline & Pheucticus tibialis & Picogrueso vientriamarillo & END & $\mathrm{I}, \mathrm{F}$ \\
\hline \multirow[t]{3}{*}{ Thraupidae } & Tangara dowii & Tangara vientricastaña & END & $\mathrm{I}, \mathrm{F}$ \\
\hline & Acanthidops bairdi & Fringilo piquiagudo & END & $N, I, F, G$ \\
\hline & Diglossa plumbea & Pinchaflor plomizo & END & $\mathrm{N}, \mathrm{I}$ \\
\hline
\end{tabular}

$R$ : residente, $M$ : migratorio, END: endémico, $R, M$ : residentes y migratorias, $A$ : accidental, $M, R$ ?: migratorio reproductivo. C: carnívoro; N: nectarívoro; F: frugívoro; I: insectívoro; O: omnívoro; G: granívoro 